\title{
The prevalence and association of major ECG abnormalities with clinical characteristics and the outcomes of real-life heart failure patients - Heart Failure Registries of the European Society of Cardiology
}

\author{
Agata Tymińska', Krzysztof Ozierański', Paweł Balsam¹, Agnieszka Kapłon-Cieślicka', Cezary Maciejewski', \\ Michał Marchel1, Maria G Crespo-Leiro², Aldo P Maggioni3,4, Jarosław Drożdż, Krzysztof J Filipiak', \\ Grzegorz Opolski', Marcin Grabowski
}

\author{
11 $1^{\text {st }}$ Department of Cardiology, Medical University of Warsaw, Warszawa, Poland \\ ¿Unidad de Insuficiencia Cardiaca Avanzada y Trasplante Cardiaco, Hospital Universitario A Coruna, CIBERCV, La Coruna, Spain \\ ${ }^{3}$ ANMCO Research Centre, Florence, Italy \\ ${ }^{4}$ EURObservational Research Programme, European Society of Cardiology, Sophia-Antipolis, France \\ ${ }^{5}$ Department of Cardiology, Medical University, Łódź, Poland
}

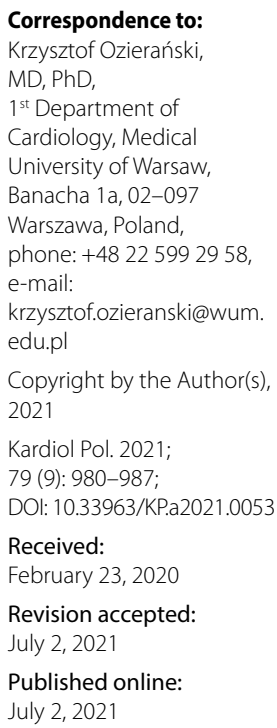

\section{A B S T R A C T}

Background: Electrocardiogram (ECG) abnormalities increase the likelihood of heart failure (HF) but have low specificity and their occurrence is multifactorial.

Aim: This study aimed to investigate the prevalence and association of major ECG abnormalities with clinical characteristics and outcomes in a large cohort of real-life HF patients enrolled in HF Registries (Pilot and Long-Term) of the European Society of Cardiology.

Methods: Standard 12-lead ECG containing at least one of the following simple parameters was considered a major abnormality: abnormal rhythm; >100 bpm; QRS $\geq 120 \mathrm{~ms}$; QTc $\geq 450 \mathrm{~ms}$; pathological Q-wave; left ventricle hypertrophy; left bundle branch block. A Cox proportional hazards regression model was used to identify predictors of the primary (all-cause death) and secondary (all-cause death or hospitalization for worsening HF) endpoints.

Results: Patients with abnormal ECG (1222/1460; 83.7\%) were older, more frequently were male and had HF with reduced ejection fraction, valvular heart disease, comorbidities, higher New York Heart Association class, or higher concentrations of natriuretic peptides as compared to those with normal ECG. In a one-year follow-up, the primary and secondary endpoints occurred more frequently in patients with abnormal ECG compared to normal ECG (13.8\% vs $8.4 \% ; P=0.021$ and $33.0 \%$ vs $24.7 \% ; P=0.016$; respectively). Abnormal rhythm, tachycardia, QRS $\geq 120 \mathrm{~ms}$, and QTC $\geq 450 \mathrm{~ms}$ were significant in univariable (both endpoints) analyses but only tachycardia remained an independent predictor of the primary endpoint. Conclusions: HF patients with major ECG abnormalities were characterized by worse clinical status and one-year outcomes. Only tachycardia was an independent predictor of all-cause death.

Key words: tachycardia, electrocardiogram, QRS duration, left bundle branch block, heart rhythm

Kardiol Pol 2021; 79, 9: 980-987

\section{INTRODUCTION}

According to the heart failure (HF) guidelines of the European Society of Cardiology (ESC), an electrocardiogram (ECG) is the basic examination that should be performed routinely in patients with suspected or known HF [1]. ECG abnormalities increase the likelihood of HF ( $89 \%$ sensitivity) but have low specificity [1]. The presence of ECG abnormalities, especially in patients with HF, may depend on many 
WHAT'S NEW?

Data from the heart failure registries of the European Society of Cardiology showed that major electrocardiogram (ECG) abnormalities (simple ECG parameters: abnormal rhythm; >100 bpm; QRS $\geq 120$ ms; QTc $\geq 450$ ms; pathological Q-wave; left ventricular hypertrophy; left bundle branch block) were present in the majority of real-life heart failure patients. What is more, these major ECG abnormalities were more common in patients with reduced left ventricular ejection fraction. Assessment of these simple major ECG abnormalities showed association with a worse general condition and one-year outcomes. Among others, tachycardia was the strongest predictor of all-cause death. Results of the ECG examination should not be overlooked as may provide important information in risk stratification.

factors (e.g. ischemia, HF etiology, electrolyte disturbances, pharmacotherapy) and is often observed. ECG abnormalities may be helpful in determining the HF etiology and making therapeutic decisions (e.g. anticoagulation in atrial fibrillation, pacing in bradycardia, cardiac resynchronization therapy [CRT] when QRS complex is prolonged). Several studies have also demonstrated that QRS duration or left bundle branch block (LBBB) can predict the risk of death in patients with chronic and decompensated HF [2-5]. Prolonged QTc (especially if genetically conditioned) may be associated with an increased risk of malignant ventricular arrhythmias, and, in selected clinical situations, may be an indication for implantation of a cardioverter-defibrillator (ICD) [6]. The importance of ECG in clinical practice is undeniable, but particularly in the chronic setting of the disease, the results of this study are often overlooked. There is also insufficient data on the prevalence and role in risk stratification of major ECG abnormalities depending on the type of $\mathrm{HF}$ - HF with reduced (HFrEF), mid-range (HFmrEF) or preserved (HFpEF) ejection fraction.

The aim of the study was to analyze the prevalence and association of easily measured major ECG abnormalities with clinical characteristics and outcomes in a large cohort of real-life HF patients enrolled in HF Registries (Pilot and Long-Term) of the ESC.

\section{METHODS}

\section{Study design}

The analysis is based on data from two HF Registries (the ESC-HF Pilot and the ESC-HF Long-Term) of the ESC. These registries were multicenter, prospective, observational surveys conducted in 136 (including 29 centers from Poland) and 211 European cardiology centers (including 35 centers from Poland), respectively. A detailed study design was previously published $[7,8]$. In short, the registries enrolled participants in outpatient and inpatient setting with chronic, worsening, or new-onset HF meeting diagnostic criteria for HF aged $\geq 18$ years. There were no other specific exclusion criteria. The study protocol was approved by local ethics committees. All participating patients were provided with detailed information and signed written consent. The electronic Case Report Form contained data on past medical history, clinical characteristics, test results, HF management, and one-year follow-up.
The current analysis concerns 2019 Polish patients. Full data on ECG recordings were available for 1611 patients, 1460 patients had available data on the primary endpoint and were included in the final analysis. The prevalence of the major ECG changes was analyzed on a standard resting 12-lead ECG. ECG containing at least one of the following parameters was considered a major abnormality: abnormal rhythm; tachycardia (>100 bpm); duration of QRS complex $\geq 120$ ms; QTc interval $\geq 450$ ms (Bazett correction); pathological Q-wave; left ventricle hypertrophy; LBBB. Patients were divided into two groups according to the presence of ECG abnormalities and compared with regard to baseline clinical characteristics, type of HF (HFrEF, HFmrEF, and HFpEF), and one-year outcomes. The type of HF was defined by the authors based on baseline LVEF measurement. The primary endpoint was all-cause death at one year. The secondary endpoint was a composite of all-cause death and hospitalization for HF worsening at one year (follow-up available for 1326 participants). The New York Heart Association (NYHA) functional class with regard to the presence of ECG changes at baseline and 12-month was also evaluated. Data regarding participants' status were collected via telephone follow-up from patients or their close relatives. If the contact was not possible, then the primary endpoint was ascertained from the data of the Polish National Health Fund.

Additionally, we sought to determine whether major ECG abnormalities were independent predictors of the primary and secondary endpoints in the study cohort.

\section{Statistical analysis}

The results were presented as median and quartiles for continuous variables and as frequencies and percentages for ordinal variables. Fisher's exact test was used for comparison of categorical variables and a Mann-Whitney $U$ test for continuous and ordinal variables. Cox proportional hazards regression models were used to identify predictors of the primary and secondary endpoints. All variables found to be statistically significant in univariable analyses $(p<0.05)$ were included in multivariable analyses. Kaplan-Meier survival curves were plotted for both study endpoints. A $P$-value below 0.05 was considered significant for all tests. All tests were two-tailed. Statistical analyses were performed using SPSS software, version 22 (IBM SPSS Statistics 22, New York, NY, USA). 
Table 1. Prevalence of major ECG abnormalities according to types of heart failure

\begin{tabular}{|c|c|c|c|c|c|}
\hline Variable & $\begin{array}{l}\% \text { of the total cohort; } \\
\text { number of patients }\end{array}$ & HFrEF $(n=806)$ & HFmrEF ( $n=279)$ & HFpEF $(n=375)$ & $P$-value \\
\hline Abnormal ECG & $83.7 \% ; 1222$ & $88.7 \% ; 715$ & $83.9 \% ; 234$ & $72.8 \% ; 273$ & $\begin{array}{c}0.14^{\mathrm{a}} \\
0.003^{\mathrm{b}} \\
<0.001^{\mathrm{c}}\end{array}$ \\
\hline Not sinus rhythm on ECG & $36 \% ; 526$ & $34.9 \% ; 281$ & $34.1 \% ; 95$ & $40 \% ; 150$ & $0.18^{\mathrm{d}}$ \\
\hline Tachycardia & $5.8 \% ; 85$ & $6 \% ; 48$ & $6.1 \% ; 17$ & $5.3 \% ; 20$ & $0.91^{d}$ \\
\hline Pathological Q-wave & $28.5 \% ; 416$ & $33 \% ; 266$ & $33 \% ; 92$ & $15.5 \% ; 58$ & $\begin{aligned} & 1.00^{\mathrm{a}} \\
< & 0.001^{\mathrm{b}} \\
< & 0.001^{\mathrm{c}}\end{aligned}$ \\
\hline LVH & $19.5 \% ; 285$ & $19.7 \% ; 159$ & $24.7 \% ; 69$ & $15.2 \% ; 57$ & $\begin{array}{c}0.27^{\mathrm{a}} \\
0.009^{\mathrm{b}} \\
0.21^{\mathrm{c}}\end{array}$ \\
\hline LBBB & $12.3 \% ; 180$ & $16.7 \% ; 135$ & $10.4 \% ; 29$ & $4.3 \% ; 16$ & $\begin{array}{c}0.042^{\mathrm{a}} \\
0.01^{\mathrm{b}} \\
<0.001^{\mathrm{c}}\end{array}$ \\
\hline QRS complex $\geq 120 \mathrm{~ms}$ & $28.1 \% ; 410$ & $37.3 \% ; 301$ & $20.1 \% ; 56$ & $14.1 \% ; 53$ & $\begin{array}{c}<0.001^{\mathrm{a}} \\
0.17^{\mathrm{b}} \\
<0.001^{\mathrm{c}}\end{array}$ \\
\hline QTc interval $\geq 450 \mathrm{~ms}$ & $44.4 \% ; 648$ & $50.5 \% ; 407$ & $35.1 \% ; 98$ & $38.1 \% ; 143$ & $\begin{array}{c}<0.001^{\mathrm{a}} \\
1.00^{\mathrm{b}} \\
<0.001^{\mathrm{c}}\end{array}$ \\
\hline
\end{tabular}

a $P$-value for HFrEF vs HFmrEF after Bonferroni correction; ${ }^{b} P$-value for HFmrEF vs HFpEF after Bonferroni correction; ${ }^{c} P$-value for $\mathrm{HFrEF}$ vs $\mathrm{HFpEF}$ after Bonferroni correction; dP-value for overall test

Abbreviations: ECG, electrocardiogram; HFmrEF, heart failure with mid-range ejection fraction; $\mathrm{HFpEF}$, heart failure with preserved ejection fraction; $\mathrm{HFrEF}$, heart failure with reduced ejection fraction; LBBB, left bundle branch block; LVH, left ventricular hypertrophy

\section{RESULTS}

\section{Prevalence of ECG abnormalities}

There were 1222 out of 1460 (83.7\%) HF patients with major ECG abnormalities. The most frequent ECG abnormalities in the entire cohort were: prolonged QTc interval (44.4\%) and abnormal heart rhythm (36.0\%). Differences in the presence of major ECG abnormalities were observed across the HF types, with the most prevalent changes observed in HFrEF patients (Table 1).

\section{Clinical characteristics}

Patients with abnormal ECG were older (median, 67.2 vs 66 years; $P=0.03$ ) and more often were male $(68.1 \%$ vs $58.8 \% ; P=0.007)$ when compared with those with normal ECG. They were also more likely to have lower left ventricular ejection fraction (median, $35 \%$ vs $45 \%$; $P<0.001$ ), moderate or severe valvular heart disease, ischemic heart disease, a history of atrial fibrillation, and chronic kidney disease than the patients with normal ECG. The group with abnormal ECG findings also had a higher NYHA class, higher concentrations of natriuretic peptides and more frequently required treatment with diuretics, antiarrhythmic (amiodarone and digitalis) and anticoagulation than the patients with normal ECG. Detailed baseline clinical characteristics of both study groups are presented in Table 2.

\section{Predictors of one-year outcomes}

In a one-year follow-up the patients with abnormal ECG were more likely to reach the primary and secondary endpoints than those with normal ECG $(13.8 \%$ vs $8.4 \%$; $P=0.021$ and $33 \%$ vs $24.7 \% ; P=0.016$; respectively) (Table
2). The Kaplan-Meier curves for the primary and secondary endpoints for both subgroups are shown in Figure 1A, B, respectively.

Across the types of HF, HFrEF patients with abnormal ECG had worse one-year outcomes when compared with the HFmrEF and HFpEF groups (Table 3, Figure 1C, D).

In the total cohort, the presence of any ECG abnormality was a predictor of both the primary and secondary endpoints but only in the univariable analyses (Supplementary material, Table S1). The univariable analyses of specific ECG abnormalities revealed abnormal rhythm, tachycardia, QRS complex duration $\geq 120 \mathrm{~ms}$, and QTc interval $\geq 450 \mathrm{~ms}$ (but not LBBB, pathological Q-wave, and left ventricular hypertrophy) to be predictors of both the primary and secondary endpoints (Supplementary material, Table S1). In the multivariable analysis, only tachycardia on ECG remained an independent predictor of the primary endpoint (hazard ratio $1.75 ; 95 \% \mathrm{Cl}, 1.06-2.87 ; P=0.03$ ) but not of the secondary endpoint (hazard ratio 1.33; 95\% Cl, 0.93-1.90; $P=0.09$ ) (Table 4).

\section{DISCUSSION}

The results of this analysis provided important epidemiological data on the prevalence, associated patients' clinical characteristics, and relevance of basic ECG abnormalities in real-life HF patients. The study showed that ECG abnormalities were present in the majority of HF patients but were observed more frequently in HFrEF patients. What is more, these easily measured ECG parameters reflected patients in worse general condition, with multiple comorbidities, and were associated with poor one-year outcomes.

A standard 12-lead ECG is an essential diagnostic tool in clinical cardiology and crucial for the management of 
Table 2. Baseline characteristics and one-year outcomes of patients with abnormal or normal ECG

\begin{tabular}{|c|c|c|c|}
\hline Variable & Abnormal ECG $(n=1222)$ & Normal ECG $(n=238)$ & P-value \\
\hline \multicolumn{4}{|l|}{ Baseline characteristics } \\
\hline Age, years & $67.2(58.2-77.0)$ & $66.0(54.8-76.6)$ & 0.04 \\
\hline Male & $68.1 \% ; 832$ & $58.8 \% ; 140$ & 0.01 \\
\hline $\mathrm{BMI}, \mathrm{kg} / \mathrm{m}^{2}$ & $27.8(25.0-31.2) ;[1169]$ & 27.8 (24.5-31.8); [227] & 0.81 \\
\hline LVEF, \% & $35(25-46)$ & $45(30-55.8)$ & $<0.001$ \\
\hline HFrEF & $58.5 \% ; 715$ & $38.2 \% ; 91$ & $<0.001$ \\
\hline HFmrEF & $19.1 \% ; 234$ & $18.9 \% ; 45$ & 0.80 \\
\hline HFpEF & $22.3 \% ; 273$ & $42.8 \% ; 102$ & $<0.001$ \\
\hline Previous HF hospitalization & $75.4 \% ; 921$ & $68.1 \% ; 162$ & 0.03 \\
\hline Coronary artery disease & $56 \% ; 684$ & $42.4 \% ; 101$ & $<0.001$ \\
\hline Prior $\mathrm{PCl}$ or $\mathrm{CABG}$ & $34.2 \% ; 417$ & $26.1 \% ; 62$ & 0.01 \\
\hline Moderate or severe mitral regurgitation & $49.7 \% ; 572 /[1150]$ & $30.7 \% ; 67 /[218]$ & $<0.001$ \\
\hline Moderate or severe aortic stenosis & $10.2 \% ; 90 /[882]$ & $4.0 \% ; 6 /[151]$ & 0.01 \\
\hline Hypertension & $63.9 \% ; 780$ & $63.0 \% ; 150$ & 0.75 \\
\hline History of atrial fibrillation & $46.5 \% ; 568$ & $21.1 \% ; 50$ & $<0.001$ \\
\hline Peripheral artery disease & $11.9 \% ; 145$ & $8.8 \% ; 21$ & 0.24 \\
\hline Diabetes & $33.6 \% ; 411$ & $27.7 \% ; 66$ & 0.20 \\
\hline Chronic kidney disease & $19.1 \% ; 233$ & $13.4 \% ; 32$ & 0.07 \\
\hline COPD & $18.3 \% ; 223$ & $15.5 \% ; 37$ & 0.44 \\
\hline Prior stroke or TIA & $12.0 \% ; 146$ & $10.1 \% ; 24$ & 0.73 \\
\hline CHA2DS2-VASc score & $4(3-5)$ & $4(2-5)$ & 0.11 \\
\hline Current malignant disease & $3.7 \% ; 45$ & $3.4 \% ; 8$ & 0.85 \\
\hline Current or former smoking & $57.9 \% ; 700$ & $56.8 \% ; 133$ & 0.71 \\
\hline Alcohol usage & $56.8 \% ; 674$ & $58.7 \% ; 135$ & 0.59 \\
\hline Pacemaker & $7.1 \% ; 87$ & $3.8 \% ; 9$ & 0.07 \\
\hline ICD & $17.4 \% ; 213$ & $12.2 \% ; 29$ & 0.13 \\
\hline CRT & $5.9 \% ; 72$ & $0.4 \% ; 1$ & $<0.001$ \\
\hline \multicolumn{4}{|l|}{ Clinical status } \\
\hline Heart rate, bpm & $80(70-96)$ & $76(68-88.2)$ & 0.01 \\
\hline $\mathrm{SBP}, \mathrm{mm} \mathrm{Hg}$ & $130(110-140)$ & $130(120-150)$ & 0.003 \\
\hline $\mathrm{DBP}, \mathrm{mm} \mathrm{Hg}$ & $80(70-83)$ & $80(70-90)$ & 0.03 \\
\hline NYHA class & $3(2-3) ;[1218]$ & $2(2-3) ;[237]$ & $<0.001$ \\
\hline Anemia & $32.4 \% ; 298 /[921]$ & $34.6 \% ; 56 /[162]$ & 0.29 \\
\hline Pleural effusion/congestion (X-ray) & $39.2 \% ; 360 /[918]$ & $27.0 \% ; 47 /[174]$ & 0.003 \\
\hline Hemoglobin, g/dl & $13.4(11.9-14.4) ;[1140]$ & 13.2 (11.1-14.4); [220] & 0.31 \\
\hline Serum creatinine, mg/dl & $1.1(0.9-1.4) ;[1158]$ & $1.0(0.8-1.3) ;[214]$ & 0.001 \\
\hline Serum sodium, mmol/l & 139 (136-141); [1157] & 139 (136-141); [220] & 0.92 \\
\hline Serum potassium, $\mathrm{mmol} / \mathrm{l}$ & $4.4(4.1-4.8) ;[1158]$ & $4.4(4.1-4.7) ;[220]$ & 0.46 \\
\hline $\mathrm{BNP}, \mathrm{pg} / \mathrm{ml}$ & 576 (203-1386.8); [163] & 364 (155-643.5); [24] & 0.01 \\
\hline NT-proBNP, pg/ml & 3048 (1352-7024); [315] & 1580 (419.5-4959.5); [55] & 0.01 \\
\hline \multicolumn{4}{|l|}{ Pharmacotherapy } \\
\hline ACE-I & 75.4\%; 921/[1221] & $75.6 \% ; 180$ & 0.93 \\
\hline ARB & $10.2 \% ; 124 /[1219]$ & $15.1 \% ; 36$ & 0.02 \\
\hline$\beta$-blocker & $89.1 \% ; 1088 /[1221]$ & $86.6 \% ; 206$ & 0.42 \\
\hline Diuretic & $83.3 \% ; 1016 /[1220]$ & $77.3 \% ; 184$ & 0.08 \\
\hline MRA & $67.3 \% ; 821 /[1221]$ & $58.6 \% ; 139$ & 0.01 \\
\hline Statins & $65.9 \% ; 805 /[1221]$ & $60.5 \% ; 144$ & 0.21 \\
\hline Oral Anticoagulant & $45.4 \% ; 554 /[1221]$ & $26.2 \% ; 62$ & $<0.001$ \\
\hline Antiplatelets & $60.0 \% ; 732 /[1221]$ & $65.5 \% ; 156$ & 0.11 \\
\hline Digitalis & $25.1 \% ; 306[1221]$ & $16.0 \% ; 38$ & $<0.001$ \\
\hline Amiodarone & $9.7 \% ; 118 /[1221]$ & $5.9 \% ; 14$ & 0.16 \\
\hline Other Antiarrhythmic & $6.1 \% ; 75 /[1221]$ & $3.4 \% ; 8$ & 0.09 \\
\hline \multicolumn{4}{|l|}{ One-year outcomes } \\
\hline NYHA class I or II & $68.2 \% 690 /[1011]$ & $81.0 \% 171 /[211]$ & $<0.001$ \\
\hline NYHA class III or IV & $31.8 \% 321 /[1011]$ & $19 \% 40 /[211]$ & \\
\hline Death & $13.8 \% ; 169$ & $8.4 \% ; 20$ & 0.02 \\
\hline Death or rehospitalization & $33 \% ; 367 /[1111]$ & $24.7 \% ; 53 /[215]$ & 0.02 \\
\hline
\end{tabular}

Continuous variables are presented as medians and interquartile ranges (IQR);

Available cases count in the respective variable are presented in square brackets.

Abbreviations: ACE-I, angiotensin-converting enzyme inhibitor; ARB, angiotensin receptor blocker; BMI, body mass index; BNP, brain natriuretic peptide; bpm, beats per minute; CABG, coronary artery bypass grafting; CHA2DS2VASc score, congestive heart failure, hypertension, age $>75$ years, diabetes mellitus, history of stroke or thromboembolism, vascular disease, age 65 to 74 years, female sex; COPD, chronic obstructive pulmonary disease; CRT, cardiac resynchronization therapy; DBP, diastolic blood pressure; HF, heart failure; ICD, implantable cardioverter-defibrillator; LVEF, left ventricular ejection fraction; MRA, mineralocorticoid receptor antagonist; NT-proBNP, N-terminal pro-brain natriuretic peptide; NYHA, New York Heart Association; PCl, percutaneous coronary intervention; SBP, systolic blood pressure; TIA, transient ischemic attack. Other — see Table 1 


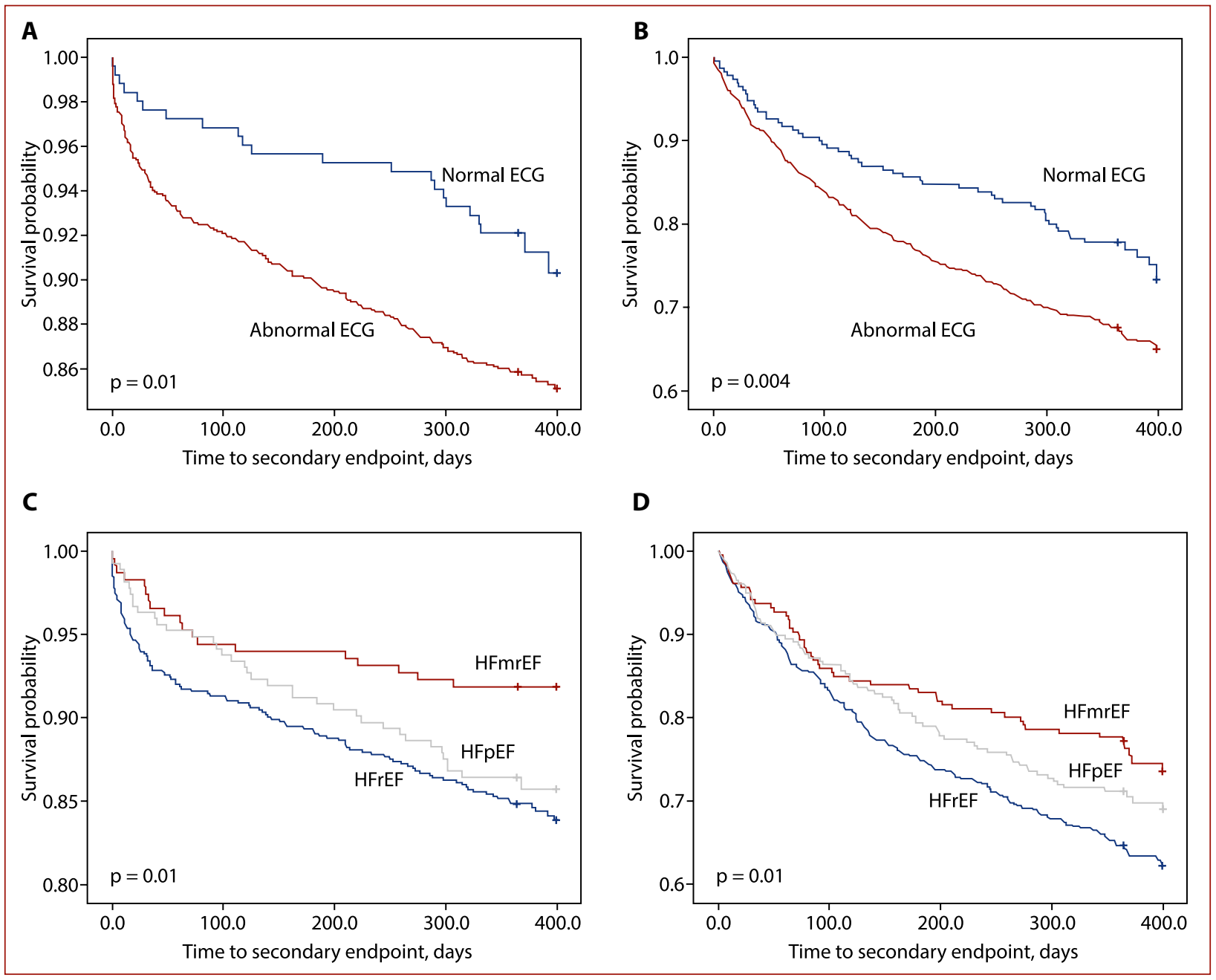

Figure 1. A. Kaplan-Meier curves for the primary endpoint of patients with abnormal or normal ECG. B. Kaplan-Meier curves for the secondary end point of patients with abnormal or normal ECG. C. Kaplan-Meier curves for the primary endpoint of patients with abnormal ECG regarding to the type of heart failure. D. Kaplan-Meier curves for the secondary endpoint of patients with abnormal ECG regarding to the type of heart failure Abbreviations: see Table 1 and 2

Table 3. One-year outcomes of patients with abnormal or normal electrocardiogram regarding to the type pf heart failure

\begin{tabular}{|c|c|c|c|}
\hline Type of HF & Abnormal ECG & Normal ECG & $P$-value \\
\hline \multicolumn{4}{|l|}{ NYHA class } \\
\hline HFrEF & $3(2-4) ; n=719$ & $2(2-3) ; n=91$ & $<0.001$ \\
\hline HFmrEF & $3(2-3) ; n=234$ & $3(2-3) ; n=45$ & $<0.001$ \\
\hline HFpEF & $3(2-3) ; n=273$ & $3(2-3) ; n=102$ & 0.20 \\
\hline \multicolumn{4}{|l|}{ Death } \\
\hline HFrEF & $15.6 \% ; 112 / 715$ & $6.5 \% ; 6 / 91$ & 0.01 \\
\hline HFmrEF & $8.1 \% ; 19 / 234$ & $6.6 \% ; 3 / 45$ & 1.00 \\
\hline HFpEF & $13.9 \% ; 38 / 273$ & $10.7 \% ; 11 / 102$ & 0.49 \\
\hline \multicolumn{4}{|c|}{ Death or rehospitalization } \\
\hline HFrEF & $36.9 \% ; 239 / 648$ & $29.6 \% ; 24 / 81$ & 0.22 \\
\hline HFmrEF & $24.8 \% ; 51 / 206$ & $26.8 \% ; 11 / 41$ & 0.84 \\
\hline HFpEF & $30 \% ; 77 / 257$ & $19.4 \% ; 18 / 93$ & 0.06 \\
\hline
\end{tabular}

Abbreviations: see Table 1 and 2

patients with most cardiovascular conditions, including patients with HF. The advantages of ECG are simplicity of implementation, non-invasive nature, low cost, and wide availability. ECG is routinely performed in most HF patients, but frequently not enough attention is paid to its results. A normal ECG is infrequently observed in patients with suspected HF but it has a low specificity [9]. Analysis of EuroHeart Failure survey data showed that ECG 
Table 4. Multivariable analysis of predictors of the primary and secondary endpoints in heart failure patients at one-year

\begin{tabular}{|c|c|c|c|c|c|c|}
\hline \multirow[t]{2}{*}{ Variable } & \multicolumn{3}{|c|}{ Primary endpoint ( $n=189$ ) } & \multicolumn{3}{|c|}{ Secondary endpoint $(n=420)$} \\
\hline & HR & $95 \% \mathrm{Cl}$ & $P$-value & HR & $95 \% \mathrm{Cl}$ & $P$-value \\
\hline Age & 1.03 & $1.02-1.05$ & $<0.001$ & 1.01 & $0.99-1.02$ & 0.20 \\
\hline Male & - & - & - & 0.77 & $0.62-0.96$ & 0.03 \\
\hline BMI & 0.98 & $0.95-1.01$ & 0.21 & - & - & - \\
\hline NYHA (class III or IV vs I or II) & 1.91 & $1.50-2.43$ & $<0.001$ & 2.02 & $1.52-2.68$ & $<0.001$ \\
\hline CKD & 1.66 & $1.19-2.34$ & 0.003 & 1.40 & $1.11-1.76$ & 0.005 \\
\hline COPD & 1.25 & $0.88-1.78$ & 0.22 & 1.20 & $0.95-1.52$ & 0.11 \\
\hline Diabetes & 1.37 & $0.98-1.93$ & 0.07 & 1.26 & $1.03-1.55$ & 0.04 \\
\hline AF history & 0.88 & $0.59-1.31$ & 0.53 & - & - & - \\
\hline $\mathrm{HGB}$ & 0.97 & $0.89-1.05$ & 0.46 & 0.96 & $0.91-1.01$ & 0.09 \\
\hline Serum sodium & 0.92 & $0.90-0.94$ & $<0.001$ & 0.96 & $0.94-0.98$ & $<0.001$ \\
\hline SBP & 1.00 & $0.99-1.01$ & 0.92 & 0.996 & $0.99-1.0001$ & 0.05 \\
\hline B-blocker & 0.47 & $0.31-0.70$ & 0.001 & 0.70 & $0.52-0.93$ & 0.01 \\
\hline ARB & 0.52 & $0.26-1.02$ & 0.06 & - & - & - \\
\hline ACE-I & 0.72 & $0.49-1.06$ & 0.1 & 0.76 & $0.61-0.95$ & 0.02 \\
\hline Abnormal rhythm & 1.08 & $0.72-1.63$ & 0.70 & 0.98 & $0.80-1.22$ & 0.88 \\
\hline Tachycardia (>100 bpm) & 1.84 & $1.12-3.03$ & 0.02 & 1.41 & $0.98-2.01$ & 0.06 \\
\hline $\mathrm{QRS} \geq 120 \mathrm{~ms}$ & 1.34 & $0.94-1.91$ & 0.11 & 1.21 & $0.97-1.51$ & 0.10 \\
\hline QTc interval $\geq 450 \mathrm{~ms}$ & 1.13 & $0.81-1.59$ & 0.46 & 1.14 & $0.92-1.41$ & 0.22 \\
\hline Digitalis & - & - & - & 1.03 & $0.82-1.30$ & 0.80 \\
\hline Amiodarone & 1.66 & $1.06-2.62$ & 0.03 & 1.19 & $0.87-1.62$ & 0.29 \\
\hline Statins & 0.76 & $0.54-1.08$ & 0.12 & - & - & - \\
\hline \multicolumn{7}{|l|}{ HF type } \\
\hline HFmrEF (reference) & 1.00 & $1.00-1.00$ & 1.00 & 1.00 & $1.00-1.00$ & 1.00 \\
\hline HFrEF & 2.04 & $1.21-3.44$ & 0.01 & 1.27 & $0.95-1.71$ & 0.10 \\
\hline HFpEF & 1.42 & $0.82-2.46$ & 0.22 & 0.86 & $0.61-1.20$ & 0.36 \\
\hline
\end{tabular}

Abbreviations: $\mathrm{ACE}-\mathrm{I}$, angiotensin converting enzyme inhibitor; $\mathrm{AF}$, atrial fibrillation; $\mathrm{ARB}$, angiotensin receptor blocker; $\mathrm{BMI}$, body mass index; bpm, beats per minute; $\mathrm{Cl}$, confidence interval; CKD, chronic kidney disease; HGB, hemoglobin; HR, hazard ratio; SBP, systolic blood pressure. Other — see Table 1 and 2

abnormalities (including ventricular rate, PR, QRS and QTC intervals, left ventriclar hypertrophy, pathological Q-wave, ST-T-wave abnormalities, and interventricular conduction abnormalities) were observed in more than $98 \%$ of $\mathrm{HF}$ patients [9]. In our study, we focused on the predefined major ECG parameters, which can be assumed as being in line with the previous observations.

All of the ECG abnormalities (except abnormal heart rhythm and tachycardia) were more prevalent in HFrEF patients when compared with patients with HFmrEF and HFpEF. Although it is known that heart rhythm abnormalities (especially atrial fibrillation) are frequently present in patients with HFpEF $[1,10]$, the presence of ECG abnormalities was more commonly associated with a history of advanced heart valve disease, atrial fibrillation, chronic kidney disease, signs of HF decompensation (higher natriuretic peptides, pleural effusion, higher NYHA class) and use of diuretics and anticoagulants.

A standard resting 12-lead ECG may reveal signs of inherited disorders but also suggests underlying structural heart disease, electrolyte disturbances and may reflect patients at higher risk of poor prognosis. In our study, the presence of at least one of the major ECG abnormalities and associated worse general condition at baseline translated into a worse prognosis in a one-year observation (higher rate of all-cause death, death, or HF hospitalization and higher NYHA class than in the patients without such ECG abnormalities). Among all tested major ECG abnormalities only tachycardia (defined as $>100 \mathrm{bpm}$ ) was an independent predictor of the primary endpoint but not the secondary endpoint. Important to note is that patients in the abnormal ECG group had significantly higher resting heart rates, despite the more frequent use of antiarrhythmic drugs (amiodarone, digitalis) and a high rate of $\beta$-blocker (89.2\%) administration. A higher resting heart rate in $\mathrm{HF}$ patients was proven to be associated with higher long-term mortality [11, 12], particularly when above $110 \mathrm{bpm}$ and with concomitant atrial fibrillation [13, 14].

It can be concluded that none of the other typical ECG parameters except tachycardia independently influenced the prognosis of patients with HF, regardless of the type of HF. However, it should also be emphasized that the presence of ECG abnormalities was a marker of patients in a worse clinical condition and with a worse one-year prognosis.

In our study, QTc prolongation was the most frequent abnormality $(44.4 \%$ and $50.5 \%$ of the whole cohort and HFrEF patients, respectively) but it was not an independent predictor of worse outcomes. Similarly, it was previously presented that QTc interval was frequently prolonged in HF patients ( $70 \%$ of patients had QTc $\geq 440 \mathrm{~ms}$ ) but it was not associated with increased long-term mortality [15].

There is evidence that both patients with reduced or mildly reduced left ventricular ejection fraction (35\%-50\%) and with LBBB are at higher risk of death and HF hospital- 
izations [16-18], and in selected cases, CRT might be particularly beneficial [1]. Similarly, QRS complex prolongation due to right ventricular pacing was shown to be associated with an increase in HF hospitalization [19]. In our study, LBBB was observed in $12.3 \%$ of the total cohort but it was not a predictor of a worse prognosis. It is worth noting that CRT was implanted in $5.8 \%$ of patients with the ECG abnormalities and nobody in the normal ECG group. It should also be highlighted that there are no standardized LBBB criteria, hence the clinical trials and registries used various and divergent definitions of LBBB. As a consequence, this translates into different observed rates of LBBB occurrence and associated prognosis [20].

Despite years of investigations, knowledge on the risk stratification for sudden cardiac death (SCD) in patients with HF is incomplete. The primary prevention of SCD is based only on left ventricular ejection fraction, which translates into difficulty in choosing patients who will benefit from ICD implantation, particularly among patients with nonischemic HF. Several ECG parameters alone or in combination were shown to have prognostic relevance (e.g. LBBB, prolonged QRS complex, prolonged QTc interval) $[6,21]$. However, despite certain clinical scenarios (CRT implantation, ICD implantation in secondary SCD prevention) they are used infrequently in risk stratification, particularly in patients with chronic $\mathrm{HF}$, or at high risk of $\mathrm{HF}$ decompensation. Therefore, further research on better risk stratification in $\mathrm{HF}$, including the risk of HF decompensation and mortality in different HF subgroups, is particularly warranted and is currently ongoing [22-24]. This might be an important strategy in guiding the HF therapy to reduce risk via further intensification of pharmacological treatment or closer monitoring [25].

\section{Limitations}

The inclusion of real-life patients followed by cardiologists is an important advantage of ESCHF Pilot and ESCHFLT registries, but drawbacks include the partial incompleteness of the data and the observational design. What is more, only the predefined ECG data in the Case Report Forms designed by the coordinators of the registries were available for analysis. The registries were not primarily focused on the ECG analysis, hence measurement errors are possible. There were no predefined definitions of the ECG parameters, including LBBB, pathological Q-wave, and left ventricular hypertrophy.

\section{CONCLUSIONS}

Results from a large real-world HF database of patients followed by cardiologists showed that ECG abnormalities were present in the majority of HF patients but more frequently in HFrEF patients. The patients with ECG abnormalities were characterized by a worse general condition and poor one-year outcomes when compared with thos without any abnormal ECG findings. Among other ECG abnormalities, only tachycardia was an independent predictor of all-cause mortality.

\section{Supplementary material}

Supplementary material is available at https://journals. viamedica.pl/kardiologia_polska.

\section{Article information}

The abstract was previously published in European Journal of Heart Failure Supplement (https://doi.org/10.1002/ejhf.1963)

Conflict of interest: None declared.

Funding: The organizational part of the registers was financed from statutory funds of the European Society of Cardiology (ESC). Investigators did not receive fees.

Open access: This article is available in open access under Creative Common Attribution-Non-Commercial-No Derivatives 4.0 International (CC BY-NC-ND 4.0) license, allowing to download articles and share them with others as long as they credit the authors and the publisher, but without permission to change them in any way or use them commercially. For commercial use, please contact the journal office at kardiologiapolska@ptkardio.pl.

How to cite: Tymińska A, Ozierański K, Balsam P, et al. The prevalence and association of major ECG abnormalities with clinical characteristics and the outcomes of real-life heart failure patients - Heart Failure Registries of the ESC. Kardiol Pol. 2021; 79(9): 980-987, doi: 10.33963/KP.a2021.0053.

\section{REFERENCES}

1. Ponikowski P, Voors A, Anker S, et al. 2016ESC Guidelines for the diagnosis and treatment of acute and chronic heart failure [in Polish]. Kardiol Pol. 2016; 74(10): 1037-1147, doi: 10.5603/kp.2016.0141.

2. Dhar R, Alsheikh-Ali AA, Estes NA, et al. Association of prolonged QRS duration with ventricular tachyarrhythmias and sudden cardiac death in the Multicenter Automatic Defibrillator Implantation Trial II (MADIT-II). Heart Rhythm. 2008; 5(6): 807-813, doi: 10.1016/j.hrthm.2008.02.013, indexed in Pubmed: 18534364.

3. Bode-Schnurbus $L$, Böcker D, Block $M$, et al. QRS duration: a simple marker for predicting cardiac mortality in ICD patients with heart failure. Heart. 2003; 89(10): 1157-1162, doi: 10.1136/heart.89.10.1157, indexed in Pubmed: 12975406.

4. Agarwal SK, Singla I, Hreybe $\mathrm{H}$, et al. Predictors of mortality amongst recipients of implantable cardioverter defibrillators for secondary prevention of cardiac arrest. Indian Pacing Electrophysiol J. 2007; 7(4): 218-224, indexed in Pubmed: 17957270.

5. Breidthardt T, Christ M, Matti M, et al. QRS and QTc interval prolongation in the prediction of long-term mortality of patients with acute destabilised heart failure. Heart. 2007; 93(9): 1093-1097, doi: 10.1136/hrt.2006.102319, indexed in Pubmed: 17395674.

6. Priori SG, Blomström-Lundqvist C, Mazzanti A, et al. ESC Scientific Document Group. 2015 ESC Guidelines for the management of patients with ventricular arrhythmias and the prevention of sudden cardiac death: The Task Force for the Management of Patients with Ventricular Arrhythmias and the Prevention of Sudden Cardiac Death of the European Society of Cardiology (ESC) Endorsed by: Association for European Paediatric and Congenital Cardiology (AEPC). Eur Heart J. 2015; 36(41): 2793-2867, doi: 10.1093/eurheartj/ehv316, indexed in Pubmed: 26320108.

7. Maggioni AP, Dahlström U, Filippatos G, et al. Heart Failure Association of the European Society of Cardiology (HFA). EURObservational Research Programme: regional differences and 1-year follow-up results of the Heart Failure Pilot Survey (ESC-HF Pilot). Eur J Heart Fail. 2013; 15(7): 808-817, doi: 10.1093/eurjhf/hft050, indexed in Pubmed: 23537547.

8. Balsam P, Ozierański K, Kapłon-Cieślicka A, et al. Differences in clinical characteristics and 1-year outcomes of hospitalized patients with heart failure in ESC-HF Pilot and ESC-HF-LT registries. Pol Arch Intern Med. 2019; 129(2): 106-116, doi: 10.20452/pamw.4418, indexed in Pubmed: 30648697. 
9. Khan NK, Goode KM, Cleland JGF, et al. EuroHeart Failure Survey Investigators. Prevalence of ECG abnormalities in an international survey of patients with suspected or confirmed heart failure at death or discharge. Eur J Heart Fail. 2007; 9(5): 491-501, doi: 10.1016/j.ejheart.2006.11.003, indexed in Pubmed: 17218150.

10. Kapłon-Cieślicka A, Tymińska A, Peller M, et al. Diagnosis, clinical course, and 1-year outcome in patients hospitalized for heart failure with preserved ejection fraction (from the Polish Cohort of the European Society of Cardiology Heart Failure Long-Term Registry). Am J Cardiol. 2016; 118(4): 535-542, doi: 10.1016/j.amjcard.2016.05.046, indexed in Pubmed: 27374606.

11. Kapłon-Cieślicka A, Balsam P, Ozierański K, et al. Resting heart rate at hospital admission and its relation to hospital outcome in patients with heart failure. Cardiol J. 2014; 21(4): 425-433, doi: 10.5603/CJ.a2013.0147, indexed in Pubmed: 24142684.

12. Laskey WK, Alomari I, Cox M, et al. AHA Get With The Guidelines ${ }^{\oplus}$-Heart Failure Program. Heart rate at hospital discharge in patients with heart failure is associated with mortality and rehospitalization. J Am Heart Assoc. 2015; 4(4): e001626, doi: 10.1161/JAHA.114.001626, indexed in Pubmed: 25904590.

13. Ozierański K, Kapłon-Cieślicka A, Balsam $P$, et al. Effect of $\beta$-blockers on 1-year survival and hospitalizations in patients with heart failure and atrial fibrillation: results from ESC-HF pilot and ESC-HF long-term registry. Pol Arch Intern Med. 2018; 128(11): 649-657, doi: 10.20452/pamw.4346, indexed in Pubmed: 30303491.

14. Simpson J, Castagno D, Doughty RN, et al. Meta-Analysis Global Group in Chronic Heart Failure (MAGGIC). Is heart rate a risk marker in patients with chronic heart failure and concomitant atrial fibrillation? Results from the MAGGIC meta-analysis. Eur J Heart Fail. 2015; 17(11): 1182-1191, doi: 10.1002/ejhf.346, indexed in Pubmed: 26358762.

15. Breidthardt T, Christ M, Matti M, et al. QRS and QTc interval prolongation in the prediction of long-term mortality of patients with acute destabilised heartfailure. Heart. 2007; 93(9): 1093-1097, doi: 10.1136/hrt.2006.102319, indexed in Pubmed: 17395674.

16. Sze E, Dunning A, Loring Z, et al. Comparison of incidence of left ventricular systolic dysfunction among patients with left bundle branch block versus those with normal QRS duration. Am J Cardiol. 2017; 120(11): 1990-1997, doi: 10.1016/j.amjcard.2017.08.003, indexed in Pubmed: 28958452.
17. Moss A, Hall W, Cannom D, et al. Cardiac-resynchronization therapy for the prevention of heart-failure events. N Engl J Med. 2009; 361(14): 1329-1338, doi: 10.1056/nejmoa0906431.

18. Witt CM, Wu G, Yang D, et al. Outcomes with left bundle branch block and mildly to moderately reduced left ventricular function. JACC Heart Fail. 2016; 4(11): 897-903, doi: 10.1016/j.jchf.2016.07.002, indexed in Pubmed: 27614941.

19. Sweeney MO, Hellkamp AS, Ellenbogen KA, et al. MOde Selection Trial Investigators. Adverse effect of ventricular pacing on heart failure and atrial fibrillation among patients with normal baseline QRS duration in a clinical trial of pacemaker therapy for sinus node dysfunction. Circulation. 2003; 107(23): 2932-2937, doi: 10.1161/01.CIR.0000072769.17295. B1, indexed in Pubmed: 12782566.

20. Jastrzębski M, Kukla P, Kisiel R, et al. Comparison of four LBBB definitions for predicting mortality in patients receiving cardiac resynchronization therapy. Ann Noninvasive Electrocardiol. 2018; 23(5): e12563, doi: 10.1111/anec.12563, indexed in Pubmed: 29806716.

21. Václavík J, Špinar J, Vindiš D, et al. ECG in patients with acute heart failure can predict in-hospital and long-term mortality. Intern Emerg Med. 2014; 9(3): 283-291, doi: 10.1007/s11739-012-0862-1, indexed in Pubmed: 23054408.

22. Jahmunah V, Oh SL, Wei JK, et al. Computer-aided diagnosis of congestive heart failure using ECG signals — a review. Phys Med. 2019; 62: 95-104, doi: 10.1016/j.ejmp.2019.05.004, indexed in Pubmed: 31153403.

23. Balsam $P$, Lodziński $P$, Tymińska $A$, et al. Study design and rationale for biomedical shirt-based electrocardiography monitoring in relevant clinical situations: ECG-shirt study. Cardiol J. 2018; 25(1): 52-59, doi: 10.5603/CJ.a2017.0102, indexed in Pubmed: 28840587.

24. Reichlin T, Abächerli R, Twerenbold R, et al. Advanced ECG in 2016: is there more than just a tracing? Swiss Med Wkly. 2016; 146: w14303, doi: 10.4414/smw.2016.14303, indexed in Pubmed: 27124801.

25. Grabowski M, Ozierański K, Balsam P, et al. The effect of sacubitril / valsartan on the occurrence of ventricular arrhythmia and the risk of sudden cardiac death in patients with chronic heart failure with reduced left ventricular ejection fraction. Expert opinion of the Heart Rhythm and Heart Failure Sections of the Polish Cardiac Society. Kardiol Pol. 2019; 77(10): 987-993, doi: 10.33963/KP.14972, indexed in Pubmed: 31527563. 